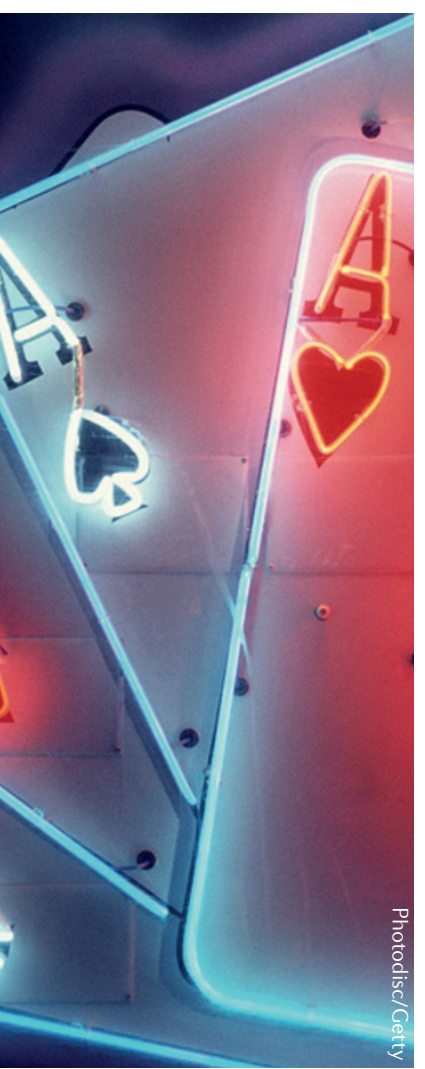

CLINICAL GENETICS

\section{DNA sequencing trumps standard screening tools}

High-throughput sequencing of fetal cell-free DNA (cfDNA) obtained from maternal plasma was found to be superior to current autosomal aneuploidy screening techniques in a recent prospective, blinded, multicentre trial of 1,914 women from the general population. "This finding will ultimately influence recommendations for prenatal screening for all pregnant women in the developed world," says lead author Diana W. Bianchi (Tufts University School of Medicine).

Bianchi et al. compared the detection of trisomy 18 and 21 using standard prenatal screening tests - measurements of maternal serum markers, such as total human chorionic gonadotropin, with or without an ultrasonography measurement of the fetal neck (which is known as the nuchal translucency test) - with that of massively parallel sequenced fetal cfDNA, which originates from placental trophoblast cells and circulates freely in maternal blood. Sequence reads obtained from cfDNA can be mapped to their chromosomes of origin and counted to calculate the normalized chromosome value, which differs in cases of aneuploidy. Outcomes were confirmed using data obtained from either physical examinations of neonates or a diagnostic cytogenetic test if available.

The predictive values of a positive screen with DNA testing were $45.5 \%$, compared with only $4.2 \%$ using the current standard screen for trisomy 21 , and $40.0 \%$ versus $8.3 \%$ for trisomy 18 . "The significantly lower false-positive results with the DNA test mean that fewer women will be made unnecessarily anxious by being told they have an abnormal test result," Bianchi believes. "As more pregnant women opt for the DNA test, we expect that fewer invasive procedures such as amniocentesis and chorionic villus sampling - which are associated with a small chance of an unintended miscarriage - will need to be performed to resolve a positive screen."

Future studies will need to address the socioeconomic implications of replacing current standard techniques with cfDNA tests as a primary non-invasive prenatal screening tool for aneuploidy.

Linda Koch

ORIGINAL RESEARCH PAPER Bianchi, D. W. et al. DNA sequencing versus standard prenatal aneuploidy screening. N. Engl.J. Med. 370, 799-808 (2014) 